\title{
Electronic structure of the self-trapped exciton in alkali fluorides and chlorides
}

\author{
K S Song $\dagger$, A M Stoneham and A H Harker \\ Theoretical Physics Division, AERE, Harwell, Didcot, Oxfordshire, OX11 ORA
}

Received 3 December 1974

\begin{abstract}
We report pseudopotential calculations for the relaxed exciton in alkali fluorides and chlorides, with emphasis on $\mathrm{NaCl}$. These calculations supplement earlier Hartree-Fock calculations by permitting investigation of a number of specific features. We have studied the more extended and higher energy excitations of the electron associated with the exciton, and we have considered a wider range of host lattices and crystal geometries.

The most important result is that the origin of the $\sigma$ (singlet) and $\pi$ (triplet) luminescence bands can be understood: the two bands derive from different orbital states, contrary to previous assumptions. Estimates of hyperfine constants, the $\sigma-\pi$ splitting and oscillator strengths are also given and agree well with experiment. The results suggest that there should be an additional $\sigma$-polarized absorption band of the self-trapped exciton in the infrared.
\end{abstract}

\section{Introduction}

The creation of an exciton in alkali halides is followed by substantial lattice distortion. The resulting centre, the self-trapped exciton, can be regarded for many purposes as an electron bound to an $X_{2}^{-}$ion, for the hole is strongly localized on two of the $X^{-}$anions. The self-trapped exciton has been the subject of much spectroscopic work, both in emission and absorption, and its nonradiative transitions play an important rôle in the radiation damage of ionic crystals.

The properties of most interest in the present paper concern the spin triplet state of the exciton, notably its spin resonance and optical absorption, and the relation between the singlet and triplet emissions associated with the self-trapped exciton. Experimentally, two luminescent bands are seen : a spin-allowed $\sigma$ band, polarized parallel to the axis of the exciton, and a spin-forbidden $\pi$ band with electric field vector normal to the axis. In some systems the $\sigma$ band is not seen, and only the $\pi$ band associated with spin triplet states is found. Qualitatively, the transitions can be understood in terms of the excitations of two halogens, $\left(\mathrm{X}^{-}\right)_{2}$. The $\sigma$ and $\pi$ bands are usually assumed to derive from the same orbital states, differing only in spin; the different polarizations occur because the spinorbit coupling is necessary to render allowed transitions from the triplet state. Quantitatively, this description fails miserably. Hartree-Fock calculations on $\mathrm{NaCl}$ (Stoneham 1974) show that the energy difference predicted is almost two orders of magnitude

$\dagger$ Permanent address : Department of Physics, University of Ottawa, Canada. 
smaller than the $2 \mathrm{eV}$ or so observed in practice. This discrepancy is far too large to result from a theoretical approximation, expecially since the same calculations give very good predictions of other transition energies. The conclusions drawn by Stoneham, which we confirm in this paper, were these. Firstly, the singlet and triplet states derive from different orbital configurations, and not from different spin states associated with the same one-electron orbitals. Secondly, since one must explain now why there is no conspicuous emission from the spin singlet state which corresponds to the ground state of the triplet exciton, it was concluded that kinetics are important too. In essence, the excitons formed in singlet states must decay radiatively or nonradiatively before reaching the lowest singlet state.

In the earlier paper, these conclusions could not be made very precise, for the orbital basis used (whilst large) was not sufficient to identify the state from which the singlet luminescence derived. In the present calculations we use a simpler approach, based on the pseudopotential method of Bartram et al (1968). The method can only treat the electronic (as opposed to hole) excitations of the exciton, but is very convenient for looking at the more extended and weakly-bound excited states. It calculates the properties of the electron component of the exciton moving in a potential determined by the lattice and the self-trapped hole. We have been able to identify the states from which the two emission bands derive, and we have been able to discuss a number of other factors, including oscillator strengths and spin resonance parameters. Whilst there are still a number of questions unresolved in detail, it is possible now to give a good qualitative and and reasonable quantitative description of the energy levels of the self-trapped exciton. Further, the methods used here are readily extended to discuss excitons involving impurity ions.

\section{The model}

In this section we discuss three basic aspects of the model : the geometry, the choice of Hamiltonian, and the form of trial functions used.

\subsection{Geometry}

The particular model we use allows us to treat all crystal ions in a point-charge approximation, and a finite number (typically the first ten shells) in a way which includes ion-size corrections. Thus the most important aspect of the geometry concerns the self-trapping distortion assumed.

Most of the calculations displace only the two halogens on which the hole is selftrapped. The separation of these ions in $\mathrm{LiF}, \mathrm{NaF}$ and $\mathrm{NaCl}$ was estimated by Stoneham (1974) by fitting optical and spin-resonance observations to predictions of $\mathrm{F}_{2}^{-}$and $\mathrm{Cl}_{2}^{-}$ properties. Other estimates are available from entirely different data (Lagendijk and Schoemaker 1975, Adrian and Jette 1974, Daly and Mieher 1969, Kabler private communication) and all give very similar values. Thus, unless otherwise stated, we should assume the halogen separations to be these:

Fluorides: $\mathrm{F}_{2}^{-}$separation $3 \cdot 75$ au

Chlorides: $\mathrm{Cl}_{2}^{-}$separation $5 \cdot 0$ au.

No dependence of this separation on the cation species is assumed, for such a dependence is believed to be small.

Some of our calculations also displace the two cations closest to the self-trapped 
exciton. Such displacements were typically $10 \%$, an amount chosen arbitrarily so that the effects of these ion motions could be discerned.

Certain of the predictions are not sensitive to the precise displacements. The order of the electronic excited states of the self-trapped exciton is hardly altered, for example. But the luminescence energies for the $\sigma$ and $\pi$ emissions appear to be very sensitive. The reason seems to be this. When the exciton recombines, the electron makes a transition from a relatively diffuse state to one which is strongly localized on two halogen ions. Thus, in the final state small movements of the ions can change the Madelung potential appreciably, so the energy of this final state varies greatly with detailed geometry. By contrast, the relatively extended states of the electron are much less sensitive to distortion. In passing, we comment that the energies of $\sigma$ and $\pi$ luminescence will not be calculated specifically in this paper, for pseudopotential methods are not suitable for calculations of the hole state; one could, of course, use two separate methods, but this introduces addition problems. However, we shall calculate the energy difference of the $\sigma$ and $\pi$ bands.

\subsection{The Hamiltonian}

The pseudopotential calculations follow the approach of Bartram et al (1968). In this method, the defect electron (here the excited electron of the exciton) moves in a potential which is the sum of the point-ion potential and an 'ion-size' correction of the form:

$$
V_{\mathrm{IS}}=\sum_{i}\left\{A_{i}+B_{i}\left(\bar{v}-U_{i}\right)\right\} \delta\left(r-\boldsymbol{R}_{i}\right) .
$$

This term is a sum over ions $i . \bar{v}$ is the expectation value of the potential energy and $U_{i}$ the point-ion potential at site $\boldsymbol{R}_{i}$. The coefficients $A_{i}$ and $B_{i}$ depend only on the species of ion.

The values of the coefficients for the alkali ions $\mathrm{M}^{+}$and halogens $\mathrm{X}^{-}$were given in the original paper by Bartram $e t$ al. In their work on the $\mathrm{F}$ centre, it was found necessary to reduce the terms $A_{i}$ by a factor $\alpha$, essentially independent of host and species. This factor, usually taken to be 0.53 , is an empirical correction for the variation of the pseudo-wavefunction over the ion cores (Gash 1970). In the present work we have usually made the same correction for the ions surrounding the self-trapped exciton.

The parameters $A_{i}$ and $B_{i}$ present more problems for the two $\mathrm{X}^{-\frac{1}{2}}$ ions on which the hole is localized. We have estimated these as the mean of the $A_{i}$ or $B_{i}$ for the two charge states $\mathrm{X}^{0}$ and $\mathrm{X}^{-}$of the halogen. In general terms, the results are that the $B_{i}$ are insensitive to the charge state. and that the $A_{i}$ are roughly the same in magnitude but opposite in sign for $\mathrm{X}^{0}$ and $\mathrm{X}^{-}$. Thus $A\left(\mathrm{X}^{-\frac{1}{2}}\right)$ is small, and it makes little difference whether any factor $\alpha$ is included or not. Values of the parameters are listed in table 1, based on the wavefunctions of Clementi (1965). Since the $\mathrm{X}_{2}^{-}$molecular ion is an open-shell system, questions arise about the spin state. We found that the difference between the exchange contributions to $A_{\mathrm{X}}$ for the singlet and triplet cases of $\mathrm{X}^{0}$ differed very little. This is consistent with the small singlet-triplet splittings given by the Hartree-Fock calculations. So in most calculations the values of $A$ used were $-10 \cdot 10 \mathrm{Ryd} a_{0}^{3}$ for $\mathrm{F}^{-\frac{1}{2}}$ and $-36 \cdot 20 \mathrm{Ryd}$ $a_{0}^{3}$ for $\mathrm{Cl}^{-\frac{1}{2}}$, prior to any correction by a factor $\alpha$.

\subsection{Wavefunction basis}

The calculations used a general computer code, PRISM, developed at Harwell by A H Harker. This code allows one to use linear combinations of Slater functions centred on the geometric centre of the exciton. One may choose to optimize the exponents in these 
Table 1. Ion-size parameters for the halogens. The results are based on Clementi's (1965) wavefunctions. For the neutral halogen, $\mathrm{X}^{0}$, the singlet and triplet exchange terms are given separately $A$ is in units Rydbergs (atomic units) ${ }^{3}$ and $B$ is in units (atomic units) ${ }^{3}$.

\begin{tabular}{lcccccccc}
\hline Ion & $A_{\mathrm{H}}$ & ${ }^{1} A_{\mathrm{X}}$ & & ${ }^{3} A_{\mathrm{X}}$ & ${ }^{1} A$ & & ${ }^{3} A$ & $B$ \\
\hline $\mathrm{F}^{0}$ & 22.236 & 7.324 & & 6.186 & 29.560 & & 28.422 & 42.68 \\
$\mathrm{~F}^{-\frac{1}{2}}$ & -10.79 & 0.97 & & 0.41 & -9.82 & & -10.38 & 46.77 \\
$\mathrm{~F}^{-1}$ & -43.81 & & -5.39 & & & -49.20 & & 50.84 \\
$\mathrm{Cl}^{0}$ & 38.234 & 5.712 & & 1.770 & 43.946 & & 40.004 & 123.856 \\
$\mathrm{Cl}^{-\frac{1}{2}}$ & -28.32 & -6.92 & & -8.88 & -35.24 & & -37.20 & 134.34 \\
$\mathrm{Cl}^{-1}$ & -94.87 & & $-19 \cdot 54$ & & & -114.41 & & 144.82 \\
$\mathrm{Br}^{0}$ & 44.374 & 2.335 & & -5.905 & -46.71 & & -38.47 & 161.91 \\
$\mathrm{Br}^{-\frac{1}{2}}$ & -36.35 & -10.84 & & -14.97 & -47.19 & & -51.32 & 176.21 \\
$\mathrm{Br}^{-1}$ & -117.82 & & -24.02 & & & -141.84 & & 190.50 \\
\hline
\end{tabular}

functions or the relative weights of the components of the linear combination. As a general rule we have tried two possibilities for each state:

(a) we have first taken a single Slater function and optimized the orbital exponent. The results make trends in behaviour particularly clear, and give guidance as to suitable exponents to take in the second method. The energies given tend to be rather high, and the convergence in the minimization is very slow.

(b) we have then taken a large number of Slater functions (typically 15) of s, p and d symmetry, and minimized the energy by adjusting the weights of the different terms. This method gives better energies and converges faster.

We shall usually be interested in the results of the second approach.

Since this is a one-electron calculation, we shall define the states by the one-electron orbital of the electron component of the exciton. The remaining electrons are in the same electronic configuration as in the ground state of the self-trapped hole. The symmetries of the one-electron states of the electron component will be given using the same notation as Stoneham (1974). Thus the totally symmetric states are $A_{1 g}$, and the states which transform in the same way as p-states are $B_{3 u}$ (parallel to the [110] axis of the exciton, say) and $\mathrm{B}_{1 u}([1 \overline{1} 0])$ and $\mathrm{B}_{2 u}([001])$ normal to the axis. We have also calculated some of the higher excited states, notably $\mathrm{A}_{1 g}^{*}$ and $\mathrm{B}_{3 \mathbf{u}}^{*}$.

\section{Energy levels for the self-trapped exciton}

We shall discuss the results in stages, so that the effects of various modifications can be determined. The effects of interest involve the trends from crystal to crystal, the flexibility of the wavefunction, the ion-size correction and the effects of lattice distortion. Results are listed in tables 2-5.

\subsection{The point-ion model}

In the point-ion model, there are two characteristic lengths : the $\mathrm{X}_{2}^{-}$separation, assumed constant for a particular halogen $\mathrm{X}$, and the host lattice parameter. Thus we expect a systematic trend from one fluoride to another, and an analogous trend in the chlorides. 
Table 2. Results for all alkali fluorides and chlorides in the point-ion approximation. The energies are in $\mathrm{eV}$. In each case the bracketed energies are for trial functions consisting of a single Slater function whose exponent was varied; the other results use many Slater functions.

\begin{tabular}{lccccc}
\hline System & $\mathrm{A}_{1 g}$ & $\mathrm{~B}_{3 u}$ & $\mathrm{~B}_{2 u}$ & $\mathrm{~B}_{1 u}$ & $\mathrm{~A}_{1 g}^{*}$ \\
\hline $\mathrm{LiF}$ & -5.228 & -4.064 & -3.038 & -2.521 & -1.517 \\
$\mathrm{NaF}$ & $(-4.687)$ & $(-3.785)$ & $(-2.655)$ & $(-2.496)$ & \\
& -4.726 & -3.948 & -2.940 & -2.352 & -2.351 \\
$\mathrm{KF}$ & $(-4.039)$ & $(-3.641)$ & $(-2.559)$ & $(-2.329)$ & \\
& -4.431 & 13.757 & -2.850 & -2.177 & -2.846 \\
$\mathrm{RbF}$ & $(-3.460)$ & $(-3.445)$ & $(-2.440)$ & $(-2.150)$ & \\
& -4.225 & -3.675 & -2.831 & -2.115 & -2.623 \\
$\mathrm{LiCl}$ & $(-3.271)$ & $(-3.368)$ & $(-2.393)$ & $(-2.086)$ & \\
& -4.325 & -3.678 & -2.952 & -2.309 & -1.908 \\
$\mathrm{NaCl}$ & $(-4.016)$ & $(-3.354)$ & $(-2.578)$ & $(-2.291)$ & \\
& -4.304 & -3.568 & -2.865 & -2.178 & -2.469 \\
$\mathrm{KCl}$ & $(-3.565)$ & $(-3.239)$ & $(-2.463)$ & $(-2.155)$ & \\
& -4.014 & -3.409 & -2.791 & -2.037 & -2.677 \\
$\mathrm{RbCl}$ & $(-3.132)$ & $(-3.089)$ & $(-2.337)$ & $(-2.008)$ & \\
& -3.610 & -3.334 & -2.765 & -1.981 & -3.115 \\
& $(-2.973)$ & $(-3.022)$ & $(-2.285)$ & $(-1.950)$ & \\
\hline
\end{tabular}

Table 3. Results for $\mathrm{NaCl}$.

\begin{tabular}{|c|c|c|c|c|c|c|c|c|c|c|}
\hline \multirow[b]{2}{*}{ Case } & \multicolumn{4}{|c|}{ Specification } & \multicolumn{6}{|l|}{ State } \\
\hline & $\alpha_{\mathrm{v}}$ & $N_{\mathrm{S}}$ & $N_{\text {B }}$ & $D$ & $\mathrm{~A}_{1 g}$ & $\mathrm{~B}_{3 u}$ & $\mathrm{~B}_{2 u}$ & $B_{1 u}$ & $\mathrm{~B}_{3 u}^{*}$ & $\mathrm{~A}_{1 g}^{*}$ \\
\hline 1 & PI & 0 & 1 & 0 & -3.565 & -3.239 & -2.463 & $-2 \cdot 155$ & & \\
\hline 2 & 0.7 & 3 & 1 & 0 & $-1 \cdot 49$ & -1.99 & $-1 \cdot 31$ & $-1 \cdot 40$ & & \\
\hline 3 & $\mathrm{PI}$ & 0 & $\mathbf{M}$ & 0 & $-4 \cdot 304$ & -3.568 & -2.865 & $-2 \cdot 178$ & & -2.469 \\
\hline 4 & $1 \cdot 32$ & 3 & $\mathbf{M}$ & 0 & $-3 \cdot 52$ & -2.73 & $-1 \cdot 39$ & $-1 \cdot 45$ & $-1 \cdot 26$ & $-1 \cdot 34$ \\
\hline 5 & $1 \cdot 32$ & 3 & $\mathbf{M}$ & 10 & $-3 \cdot 28$ & $-2 \cdot 61$ & $-1 \cdot 38$ & -1.44 & -1.27 & -1.34 \\
\hline 6 & $1 \cdot 32$ & 10 & $\mathrm{M}$ & 10 & $-3 \cdot 26$ & $-2 \cdot 50$ & -0.80 & -0.82 & -0.80 & -0.53 \\
\hline 7 & $1 \cdot 32$ & 10 & $\mathbf{M}^{\prime}$ & 10 & $-3 \cdot 15$ & $-2 \cdot 04$ & -0.84 & -0.84 & $-0 \cdot 81$ & -0.66 \\
\hline 8 & 1.0 & 10 & $\mathbf{M}^{\prime \prime}$ & 10 & $-3 \cdot 132$ & $-2 \cdot 019$ & -0.842 & -0.841 & -0.814 & -0.654 \\
\hline 9 & 1.0 & 10 & $\mathbf{M}^{\prime \prime \prime}$ & 10 & $-3 \cdot 181$ & -2.439 & -0.817 & -0.818 & -0.767 & -0.432 \\
\hline 10 & $1 \cdot 0$ & 10 & $\mathbf{M}^{\prime \prime \prime \prime}$ & 10 & -2.434 & & & -0.827 & & -0.814 \\
\hline 11 & 0.53 & 10 & $\mathbf{M}^{\prime \prime}$ & 10 & $-3 \cdot 121$ & -1.995 & -0.842 & -0.841 & -0.813 & -0.651 \\
\hline 12 & 0.53 & 10 & $\mathbf{M}^{\prime \prime \prime}$ & 10 & $-3 \cdot 154$ & $-2 \cdot 391$ & -0.817 & -0.818 & -0.764 & -0.426 \\
\hline 13 & 0.53 & 10 & $\mathbf{M}^{\prime \prime \prime \prime}$ & 10 & $-2 \cdot 390$ & & & -0.827 & & -0.814 \\
\hline 14 & 0.53 & 10 & $\mathbf{M}^{v}$ & 10 & -2.992 & & & -0.787 & & -0.847 \\
\hline
\end{tabular}

$\alpha_{\mathrm{H}}=\alpha$ for ions other than $V_{\mathrm{K}}$ ions $=0.53$ in all cases. $\alpha_{\mathrm{V}}=\alpha$ for the two $\mathrm{X}^{-\frac{1}{2}}$ ions; PI means point-ion approximation. $N_{\mathrm{S}}=$ number of shells with ion-size correction. $N_{\mathrm{B}}=1$ when only one Slater function, and $=\mathbf{M}$ when many functions. Here $\mathbf{M}, \mathbf{M}^{\prime}, \mathbf{M}^{\prime \prime}$ indicate different choices in detail. The exponents are in ratios $\mathbf{K}\left(\mathbf{M}^{\prime \prime \prime}\right): \mathbf{K}\left(\mathbf{M}^{\prime \prime}\right): \mathbf{K}\left(\mathbf{M}^{\prime}\right)=1 \cdot 3: 0 \cdot 7: 1 \cdot 0 . \quad D=\%$ outward displacement of the two nearest cations.

This is what is found, and is seen most clearly from the results using a single Slater function with variable exponent. As the lattice parameter increases, the energy levels rise, so that the energies for transitions between excited states of the exciton fall. At the same time, the wavefunctions become more extended. The trend is very similar to that for $\mathrm{F}$ centres in the same hosts. To give a rough order-of-magnitude, for the lowest $\mathrm{A}_{1 \mathrm{~g}}$ 
Table 4. Results for $\mathrm{NaF}$.

\begin{tabular}{|c|c|c|c|c|c|c|c|c|c|c|}
\hline \multirow[b]{2}{*}{ Case } & \multicolumn{4}{|c|}{ Specification } & \multicolumn{6}{|l|}{ State } \\
\hline & $\alpha_{\mathbf{Y}}$ & $N_{\mathrm{S}}$ & $N_{\mathrm{B}}$ & $D$ & $A_{1 g}$ & $\mathbf{B}_{3 u}$ & $\mathrm{~B}_{2 u}$ & $\mathbf{B}_{1 u}$ & $\mathrm{~B}_{3 u}$ & $A_{1 g}^{*}$ \\
\hline 1 & PI & 0 & 1 & 0 & -4.039 & -3.641 & -2.559 & $-2 \cdot 329$ & & \\
\hline 2 & PI & 0 & $\mathbf{M}$ & 0 & $-4 \cdot 726$ & -3.948 & $-2 \cdot 940$ & $-2 \cdot 352$ & & $-2 \cdot 351$ \\
\hline 3 & $1 \cdot 0$ & 10 & $\mathbf{M}$ & 14 & $-3 \cdot 113$ & $-2 \cdot 296$ & -0.919 & -1.020 & -0.894 & -0.326 \\
\hline 4 & 1.0 & 10 & $\mathbf{M}^{\prime}$ & 14 & -3.289 & & & -1.033 & & +0.497 \\
\hline 5 & 0.53 & 10 & $\mathrm{M}$ & 14 & -3.086 & $-2 \cdot 251$ & -0.919 & -1.020 & -0.888 & -0.324 \\
\hline 6 & 0.53 & 10 & $\mathbf{M}^{\prime}$ & 14 & $-3 \cdot 271$ & & & -0.928 & & $+1 \cdot 168$ \\
\hline 7 & 0.53 & 10 & $\mathbf{M}^{\prime \prime}$ & $\mathrm{k} 4$ & $-2 \cdot 328$ & & & -0.951 & & -1.006 \\
\hline
\end{tabular}

The notation is as in table 3 , except that here $\mathrm{K}(\mathrm{M}): \mathrm{K}(\mathrm{M})=0 \cdot 7: 1 \cdot 0$.

Table 5. Spectroscopy of the relaxed exciton in $\mathrm{NaCl}$.

\begin{tabular}{clll}
\hline Transition & $\begin{array}{l}\text { Hartree-Fock } \\
\text { theory (Stoneham 1974) }\end{array}$ & $\begin{array}{l}\text { Experiment } \\
\text { (Williams and Kabler 1974) }\end{array}$ & $\begin{array}{l}\text { Pseudopotential } \\
\text { (present paper) }\end{array}$ \\
\hline $\begin{array}{l}\text { lonization } \\
\text { M-like: }\end{array}$ & $3 \cdot 16$ & $2 \cdot 8$ & Not calculated \\
$\mathrm{A}_{1 g} \leftrightarrow \mathrm{B}_{2 u}$ & Not calculated & & $2 \cdot 3-2 \cdot 4[0 \cdot 24]$ \\
$\mathrm{A}_{1 g} \leftrightarrow \mathrm{B}_{1 u}$ & 1.49 & $2 \cdot 1$ & $2 \cdot 3-2 \cdot 4[0 \cdot 26]$ \\
$\mathrm{A}_{1 g} \leftrightarrow \mathrm{B}_{3 u}^{*}$ & Not found & & $2 \cdot 3-2 \cdot 5[0 \cdot 02]$ \\
$\begin{array}{c}\text { Other: } \\
\mathrm{A}_{1 g} \leftrightarrow \mathrm{B}_{3 u}\end{array}$ & Not found & Not seen & $0 \cdot 7-1 \cdot 1[0 \cdot 50]$ \\
\hline
\end{tabular}

The transition energies are in $\mathrm{eV}$. The oscillator strengths for the pseudopotential case are given in square brackets [ ]; for $A_{1 g} \leftrightarrow B_{3 \psi}, B_{3 t}^{*}$ the results are sensitive to factors like choice of basis. The range of values indicates the model-dependence.

Table 6. Spectroscopy of the relaxed exciton in $\mathrm{NaF}$.

\begin{tabular}{|c|c|c|c|}
\hline Transition & $\begin{array}{l}\text { Hartree-Fock } \\
\text { theory (Stoneham 1975) }\end{array}$ & Experiment & $\begin{array}{l}\text { Pseudopotential } \\
\text { (present paper) }\end{array}$ \\
\hline $\begin{array}{l}\text { Ionization } \\
\text { M-like : }\end{array}$ & 3.63 & Not seen & Not calculated \\
\hline $\begin{array}{l}\mathrm{A}_{1 g} \leftrightarrow \mathrm{B}_{2 u} \\
\mathrm{~A}_{1 g} \leftrightarrow \mathrm{B}_{1 u} \\
\mathrm{~A}_{1 g} \leftrightarrow \mathrm{B}_{3 \psi} \\
\text { Other: }\end{array}$ & Not calculated & Not seen; $\mathrm{M}$ band at $2.47 \mathrm{eV}$ & $\begin{array}{l}2 \cdot 2 \\
2 \cdot 1-2 \cdot 2 \\
2 \cdot 2-2 \cdot 25\end{array}$ \\
\hline $\mathrm{A}_{1 \theta} \leftrightarrow \mathrm{B}_{3 \mu}$ & 0.35 & Not seen & 0.82 \\
\hline
\end{tabular}

state, the mean radius $\langle r\rangle$ and lattice parameter are roughly proportional:

$$
\begin{array}{ll}
\langle r\rangle \simeq 1.23 a & \text { (fluorides) } \\
\langle r\rangle \simeq 1.13 a & \text { (chlorides) }
\end{array}
$$

When a large number of radial functions is used (typically 15 for the $\mathrm{A}_{1 g}$ states and 6 for the others), the conclusions are qualitatively similar, although the energies are significantly lower. The $\mathrm{A}_{1 g}$ state is lowest, as expected. $\mathrm{A}_{3 u}$ state lies a mere $0.6-0.8 \mathrm{eV}$ higher 
in energy, with $\mathrm{B}_{2 u}$ and $\mathrm{B}_{1 u}$ states lying around $2 \mathrm{eV}$ above the $\mathrm{A}_{1 g}$ state. Even at this stage, there is fair agreement with experiment, for the $\mathrm{A}_{1 q}-\mathrm{B}_{1 u}$ and $\mathrm{A}_{1 q}-\mathrm{B}_{2 u}$ transitions are the $\pi$-polarized M-like transitions seen by Williams and Kabler (1974). Further, the point-ion model correctly predicts the trend with lattice parameter seen by these workers. We shall argue later that the $A_{1 g}-B_{3 u}$ transition has not been found yet although it should be observable; it is not the M-like transition reported by Williams and Kabler, which is $\mathrm{A}_{1 g}-\mathrm{B}_{3 u}^{*}$ in our notation. We also argue that the $\mathrm{A}_{1 g}^{*}$ state, located about $2 \mathrm{eV}$ above $\mathrm{A}_{1 g}$ in most cases, is the source of the $\sigma$ emission.

Problems can arise in the point-ion model because the cations are so very attractive, favouring too-anisotropic functions. An example can be seen for $\mathrm{RbF}$ and $\mathrm{RbCl}$, where the one-Slater $A_{1 g}$ state lies above the corresponding $B_{3 u}$ state. Even the simplest ionsize corrections eliminate these anomalies.

\subsection{Effects of finite ion-size, extended basis and distortion}

We need to study two separate ion-size corrections: those concerned with the two $X^{-\frac{1}{2}}$ ions, and those concerned with the anions and cations of the 'perfect' host lattice outside. Since many of the wavefunctions we shall consider are comparable in extent with an $\mathrm{F}$ centre, it is reasonable to follow Bartram et al (1968) in reducing the $A$ for the host ions by the empirical factor $\alpha=0.53$. Various possibilities are open for the $X^{-\frac{1}{2}}$ ions, for the rate of change of the wavefunction over their cores is different from that for the $X^{-}$, leading to other values of $\alpha$. We denote by $\alpha_{H}$ and $\alpha_{V}$ the values of $\alpha$ for the host and $V_{K}$ ions respectively.

3.2.1. Effects of ion size. The effects can be seen both from the single-Slater data for $\mathrm{NaCl}$ and from the $\mathrm{NaF}$ and $\mathrm{NaCl}$ calculations with more extended basis.

Qualitatively, all results show that the energies with ion-size corrections are less negative and the orbits somewhat more extended than the results for the point-ion model. This occurs because the ion-size corrections are repulsive on the attractive cations. The order of the energy levels is as for the point-ion case $\left(\mathrm{A}_{1 g}<\mathrm{B}_{3 u}<\mathrm{B}_{2 u}<\right.$ $\left.\mathrm{B}_{1 u} \leqslant \mathrm{~B}_{3 u}^{*} \leqslant \mathrm{~A}_{1 g}^{*}\right)$ except for the single-Slater case, where the $\mathrm{A}_{1 g}$ state is represented badly, and lies above $\mathrm{B}_{3 u}$.

The extent of the cancellation between the $A$ and $B$ terms in equation (2.1) is sensitive to $\alpha_{\mathrm{v}}$, the values of $\alpha_{\mathrm{v}} \sim 1.3$ being closest to the point-ion results. However, the results for the energy splittings are much less sensitive to $\alpha_{\mathrm{v}}$. We discuss later the splittings and their comparison with experiment and earlier Hartree-Fock values.

The highly-excited states prove relatively extended, and it is necessary to include ion-size corrections for at least ten shells; the lowest $\mathrm{A}_{1 g}$ and $\mathrm{B}_{3 u}$ states are fairly compact and the differences between three and ten shells are not important.

3.2.2. Effects of choice of basis. As mentioned earlier, the lowest $A_{1 g}$ state is represented badly by a single Slater function. But even with 15 Slater functions, the absolute energy levels are sensitive to the precise basis chosen. This sensitivity is most important for the lowest $\mathrm{A}_{1 g}$ state, the higher-energy $\mathrm{B}_{2 u}, \mathrm{~B}_{1 u}, \mathrm{~B}_{3 u}^{*}$ and $\mathrm{A}_{1 g}^{*}$ states being roughly independent of basis. Unfortunate $y$, the $A_{1 g}-A_{1 g}^{*}$ separation varies markedly with basis, ranging from $1.47 \mathrm{eV}$ to $2.74 \mathrm{eV}$ for $\mathrm{NaCl}$. Since these two states have the same symmetry, one cannot merely select the lowest energy for each state separately. There is also a technical problem concerning orthogonality. In the point-ion model, $\mathrm{A}_{1 g}$ and $\mathrm{A}_{1_{g}}^{*}$ are orthogonal. But, with the ion-size corrections, the Hamiltonian depends on the state through $\bar{v}$ in equation 
(2.1), and strict orthogonality is not guaranteed. Fortunately, the ion-size corrections are small for $\mathrm{NaCl}$ and $\mathrm{NaF}$, and the problem has no consequences of real significance. But care is necessary in other systems involving larger ions. Most of the sets of results for $\mathrm{NaCl}$ give $\mathrm{A}_{1 g}-\mathrm{A}_{1 g}^{*}$ separations in the range $2 \cdot 15-2.75 \mathrm{eV}$, centring on $2.45 \mathrm{eV}$, and we shall assume this is a good value. The corresponding value for $\mathrm{NaF}$ is around $2.75 \mathrm{eV}$.

3.2.3. Effects of lattice distortion. It is likely that the cations nearest to the two $X^{-\frac{1}{2}}$ ions are displaced outwards as the two halogens are drawn together. This follows from both theoretical (Jette et al 1969) and experimental (Daly and Mieher 1969) work on the $V_{K}$ centre. We have usually assumed a $14 \%$ outward displacement for $\mathrm{NaF}$ and a $10 \%$ displacement for $\mathrm{NaCl}$.

The distortion has very little effect. The lowest electron levels $\left(\mathrm{A}_{1 g}\right.$ and $\left.\mathrm{B}_{3 w}\right)$ are raised by about $0.1 \mathrm{eV}$ as the attractive cations move away; the higher states are unaffected. However, as mentioned in $\$ 2.1$, the energy of the state to which recombination occurs does vary with geometry. As a result, the energy of the recombination luminescence is very sensitive to distortion.

\subsection{Summary of results}

It is useful to summarize the results of these calculations to point out the common features. The notable points are these, irrespective of model, distortion, or basis :

(a) the order of the

$$
\mathrm{A}_{1 g}<\mathrm{B}_{3 u}<\mathrm{B}_{2 u}<\mathrm{B}_{1 u} \leqslant \mathrm{~B}_{3 u}^{*}<\mathrm{A}_{1 g}^{*}
$$

and this order is only violated in rare special cases not of immediate concern here.

(b) the $\mathrm{A}_{1 g}$ and $\mathrm{B}_{3 u}$ levels are relatively compact, roughly similar in extent to an $\mathrm{F}$ centre. In consequence their properties are sensitive to the choice of basis, distortion and $\alpha_{\mathrm{V}}$, but not to the number of shells for which the ion-size correction is applied.

(c) the higher states $\left(\mathrm{B}_{2 u}, \mathrm{~B}_{1 u}, \mathrm{~B}_{3 u}^{*}\right.$ and $\left.\mathrm{A}_{1 g}^{*}\right)$ are relatively diffuse, with $\langle r\rangle$ three or four times larger than for the lower states. The choices of local lattice distortion and $\alpha_{\mathrm{V}}$ are not important, nor is the choice of basis critical except possibly for $\mathrm{A}_{1 g}^{*}$. On the other hand, one must include ion-size corrections out to at least ten shells.

These features hold for $\mathrm{NaCl}, \mathrm{NaF}$ and for the less-detailed calculations on other systems.

\section{Discussion: spectroscopy of the self-trapped exciton}

We now relate the results of $\S 3$ to experiment and with earlier theory. For simplicity, in defining the exciton states formed from the hole in its lowest state and the electron in an orbital of symmetry $\Gamma$, we shall write ${ }^{\mathrm{T}} \Gamma$ for the triplet exciton and ${ }^{\mathrm{S}} \Gamma$ for the corresponding singlet. The essential clue in understanding the observations is this: the longlived state which gives rise to the $\pi$-luminescence is ${ }^{\mathrm{T}} \mathrm{A}_{1 g}$, and the short-lived state giving $\sigma$-luminescence is ${ }^{\mathrm{s}} \mathrm{A}_{1 g}^{*}$. The recombination occurs from a different orbital state in each case. This is a new feature, for the standard assumption has always been that recombination has been from a single orbital state, for example, that the $\pi$ - and $\sigma$-luminescence derive from ${ }^{\mathrm{T}} \mathrm{A}_{1 g}$ and ${ }^{\mathrm{S}} \mathrm{A}_{1 g}$ respectively. 


\subsection{Spectroscopy of the triplet exciton}

We begin by discussing the allowed electronic transitions of the ${ }^{\mathrm{T}} \mathrm{A}_{1 g}$ state, since this allows a check of our work by comparison with theory and experiment. The results for $\mathrm{NaCl}$ are summarized in table 5 . It can be seen that there are four allowed transitions. Three (to the $\mathrm{B}_{2 u}, \mathrm{~B}_{1 u}$ and $\mathrm{B}_{3 u}^{*}$ states) correspond closely in energy to the M-like transitions of Kabler and Williams. There is also an extra transition to $\mathrm{B}_{3 w}$ with much lower energy but with a significant oscillator strength. This extra transition has not been seen yet. Its energy is somewhat model-dependent.

For $\mathrm{NaF}$, similar results are found, with M-like transitions predicted at about $2 \cdot 2 \mathrm{eV}$ plus a $\sigma$-polarized transition at about $0.8 \mathrm{eV}$. The oscillator strength for this lower-energy transition is around $0 \cdot 23$, similar to the value for the $\pi$-polarized M-like transitions. Hartree-Fock calculations also show a low-energy $\sigma$ excitation (A M Stoneham unpublished). No experimental data are available for $\mathrm{NaF}$.

The results for $\mathrm{NaCl}$ show very satisfying consistency. The agreement of experiment and theory is at least as good as one would expect for such complicated systems. The differences between the Hartree-Fock and pseudopotential results have several obvious sources. One is the lattice distortion, since the nearest cations were moved in the present calculations. A second difference is the number of point-ions included : the present work takes an infinite crystal, whereas Stoneham (1974) treated only 48 sites. The differences in degree of self-consistency and in basis are other factors. Given so many differences, the results are acceptably close.

\subsection{The $\sigma$-luminescence}

We now turn to the evidence that the singlet $\sigma$-luminescence involves recombination from ${ }^{\mathrm{s}} \mathrm{A}_{1 g}^{*}$ rather than ${ }^{\mathrm{s}} \mathrm{A}_{1 g}$. The arguments are these. Firstly, the ${ }^{\mathrm{T}} \mathrm{A}_{1 g}$ and ${ }^{\mathrm{s}} \mathrm{A}_{1 g}$ states differ in energy by a few hundredths of an $\mathrm{eV}$. This is almost two orders of magnitude less than that observed, and the difference cannot be resolved by changes in geometry or basis. But ${ }^{\mathrm{s}} \mathrm{A}_{1 g}^{*}$ and ${ }^{\mathrm{T}} \mathrm{A}_{1 g}$ differ in energy by about the right amount $(1.6$ to $2.7 \mathrm{eV}$ in $\mathrm{NaCl}$, depending on the model, of $2.0 \mathrm{eV}$ observed) and both states have the correct spin and symmetry. Secondly, as Blair et al (1972) have argued, the dipole matrix elements for the $\sigma$ and $\pi$ transitions appear to be inconsistent if one assumes they both refer to the same orbital state. Finally, Kabler (1974) has argued that this work with Williams on intersystem crossing also favours an interpretation in terms of two distinct orbital states.

One obvious question follows: if the $\sigma$ luminescence derives from ${ }^{\mathrm{s}} \mathrm{A}_{1 g}^{*}$, why is no luminescence seen from ${ }^{\mathrm{s}} \mathrm{A}_{1 g}$, the lower singlet state? The answer (see eg Stoneham 1974 \$3.2) presumably lies in kinetics, rather than energetics. When an exciton is formed in a singlet state, as it evolves there must be a high probability of recombination ( $\epsilon$ ither radiative or nonradiative) before the ${ }^{\mathrm{s}} \mathrm{A}_{1 g}$ state is reached. But when the exciton is formed as a spin triplet, radiative recombination is spin-forbidden in the absence of spin-orbit coupling, so that the exciton is able to reach the lowest orbital state, ${ }^{\mathrm{T}} \mathrm{A}_{1 \mathrm{~g}}$, prior to luminescing.

Two deductions can be made if this is the explanation. First, there will be a variation from system to system of the nonradiative and other processes which lead to luminescence from ${ }^{s} A_{1 g}^{*}$ rather than ${ }^{s} A_{1 g}$. It would not be surprising if, in some systems, nonradiative processes prevent ${ }^{\mathrm{s}} \mathrm{A}_{1 g}^{*}$, as well as ${ }^{\mathrm{s}} \mathrm{A}_{1 g}$, being populated. Thus one might expect to find $\pi$-luminescence in all cases, but $\sigma$-luminescence only in some. This is observed : neither $\mathrm{KCl}$ or $\mathrm{RbCl}$ appear to show $\sigma$-luminescence, for example. Secondly, if one could popu- 
late the ${ }^{\mathrm{S}} \mathrm{A}_{1 g}$ state, it should luminesce. Since ${ }^{\mathrm{T}} \mathrm{A}_{1 g}$ has a long lifetime, it might be possible to populate ${ }^{\mathrm{S}} \mathrm{A}_{1 g}$ thermally. This would lead to a temperature-dependence of the degree of polarization and of the lifetime. Pooley and Runciman (1970 \$3.4) observe a different temperature-dependence of lifetime and luminescent intensity in $\mathrm{KCl}$ which may be relevant; the same phenomenon may occur in $\mathrm{KBr}$. There have also been some recent measurements on $\mathrm{KCl}$ (Purdy and Murray 1974) which indicate a state at about the right position above ${ }^{\mathrm{T}} \mathrm{A}_{1 g}$, although the observed behaviour is more complicated than suggested above. But the result is sufficiently encouraging that the effect should be sought in other systems.

\subsection{Spin resonance and transition probabilities}

So far we have been concerned primarily with the energies of the states. We now verify that the wavefunction predictions are also satisfactory.

4.3.1. Dipole matrix elements. Blair et al (1972) have estimated that the dipole matrix element for the $\sigma$ transition is about $0.61 \AA$. This is strictly $\left(E_{\text {eff }} / E_{0}\right)|\langle i|r| \mathrm{f}\rangle|$, where $\left(E_{\text {eff }} / E_{0}\right)$ is the effective-field correction, and $|\mathrm{i}\rangle$ and $|\mathrm{f}\rangle$ are the initial and final states. We have estimated $\mid\left\langle\mathrm{A}_{1 g}^{*}\left|\boldsymbol{r} \mathrm{B}_{3 u}^{0}\right|\right\rangle$, where $\mathrm{B}_{3 u}^{0}$ is an LCAO approximation to the hole state to which recombination occurs. The result is sensitive to the detailed assumptions, for there can be cancellation between the various components of the $\mathrm{A}_{1 g}^{*}$ wavefunction. For the case we believe most plausible (case 8 of table 3) we find

$$
\left|\left\langle\mathrm{A}_{1 g}^{*}|\boldsymbol{r}| \mathrm{B}_{3 u}^{0}\right\rangle\right|=0.32 \AA \text {. }
$$

Even without an effective-field correction, this is satisfactorily close to observation. With an effective-field correction of about a factor 2 (typical of many effective-mass and compact systems : see the surveys by Smith and Dexter 1973, Stoneham 1975) the result would be still more satisfactory,

4.3.2. The isotropic hyperfine constants. Spin resonance has been observed for triplet self-trapped excitons in $\mathrm{NaF}$ (Call 1974) and $\mathrm{NaCl}$ (Wasiela et al 1973). The zero-field splitting has been obtained in both cases, although hyperfine constants have been obtained only for $\mathrm{NaF}$. Call has measured the axial hyperfine constant on the central fluorines as 395 gauss, and finds a linewidth (presumably due to hyperfine interactions with other fluorines) of 160 gauss. The hyperfine constant on the central ions is dominated by the hole wavefunction, and is not really a test of the present work. However, the electron part of the wavefunction dominates in the linewidth, where the standard formulae (eg Kittel 1954 or equation (5.8) of Stoneham 1969) are applicable.

We have calculated the isotropic hyperfine constants by orthogonalizing the electron pseudowavefunction to the ion cores using the sopHIC program developed by $\mathrm{A} \mathrm{H}$ Harker. The ion cores were symmetrically-orthogonalized to each other (cf Harker 1974). The linewidths predicted (omitting contributions from the central ions) are 126 gauss for $\mathrm{NaF}$ and 80 gauss for $\mathrm{NaCl}$. The electron contribution to the isotropic constants for the central ions is 72 gauss for $\mathrm{NaF}$ and 20 gauss for $\mathrm{NaCl}$, assuming ${ }^{35} \mathrm{Cl}$. The predictions are of the isotropic constants, whereas the measurements also involve the anisotropic constants. However, it is clear that the results agree well with experiment. 


\section{Conclusions}

The model proposed here is in qualitative and quantitative agreement with all available experimental data on the self-trapped excitons in alkali halides. Successful predictions include nine different energy splittings (including results from our earlier Hartree-Fock work), electric-dipole matrix elements and hyperfine constants.

The main new feature of our model is the recognition that different electronic configurations are responsible for the singlet and triplet luminescence bands. This assumption appears to remove all the significant problems of earlier models, and suggests that the main aspects of the self-trapped exciton are now satisfactorily understood.

\section{Acknowledgments}

We are indebted to Dr A E Hughes, Dr D Pooley, Dr L Hobbs and Dr M N Kabler for stimulating discussions and comments. One of us (KSS) would like to thank the Theoretical Physics Division, Harwell, for its hospitality during the period in which this work was done.

\section{References}

Bartram R H, Stoneham A M and Gash P 1968 Phys. Rev. 176101424

Blair I M, Pooley D and Smith D 1972 J. Phys. C: Solid St. Phys. 5 1537--52

Call P 1974 D.Phil. Thesis University of Oxford

Clementi E 1965 IBM J. Res. Dev. 9 suppl.

Daly D F and Mieher R L 1969 Phys. Rev. 183 368-83

Gash P 1970 PhD Thesis University of Connecticut

Harker A H 1974 J. Phys. C: Solid St. Phys. 7 3224-38

Jette A N, Gilbert T L and Das T P 1969 Phys. Rev. $184884-94$

Kabler M N 1974 Colour Centre Conference, Sendai abstract C15

Kittel C 1954 Defects in Crystalline Solids (London: The Physical Society) pp 33-7

Pooley D and Runciman W 1970 J. Phys. C: Solid St. Phys. 3 1815-24

Purdy A E and Murray R B 1974 Colour Centre Conference, Sendai abstract D39

Smith D Y and Dexter D L 1973 Prog. Opt. 10107

Stoneham A M 1969 Rev. Mod. Phys. 41 82-108

1974 J. Phys. C: Solid St. Phys. 7 2476-86

1975 Theory of Defects in Solids (London: Oxford UP) $\$ 10.5$

Wasiela A, Ascarelli G and Merle d'Aubigne Y 1973 J. Phys. Paris, suppl. 34 C9 123-30

Williams R T and Kabler 1974 Phys. Rev. B9 1897-908 